\title{
Détermination des propriétés élastiques d'éléments individuels du plan ligneux du chêne par des essais de traction sur micro-éprouvettes
}

\author{
Éric Badel, Patrick Perré* \\ Équipe produits forestiers, Engref-Inra, 14, rue Girardet 54042 Nancy cedex, France
}

(Received 22 July 1998; accepted 3 December 1998)

\begin{abstract}
Determination of the elastic properties using tensile tests on micro-samples for different anatomical elements for oak. This work proposes elastic values measured in the cross section of wood for different anatomical elements of oak. The experimental device, conceived and developed in our laboratory, allows the Young's modulus and Poisson's ratio to be determined on very small samples. In this work, four components of the anatomical pattern have been differentiated. The corresponding micro samples have been prepared by careful polishing, which is difficult at best. Finally, we are able to propose values for ray cells, longitudinal parenchyma cells, initial ring porous zone and fibres. The ray cells depict the highest rigidity in the transverse plane. On the contrary, the ring porous zone is the weakest, with a value almost ten times less important. Fibres and longitudinal parenchyma cells exhibit average values. The later only seem to present a high anisotropy ratio $\left(\mathrm{E}_{\mathrm{R}} / \mathrm{E}_{\mathrm{T}} \approx 2\right)$. (@ Inra/Elsevier, Paris.)
\end{abstract}

elastic properties / anatomy / oak / microscopic / experiment

Résumé - Ce travail propose des valeurs de propriétés élastiques mesurées dans le plan transverse du chêne pour différents éléments du plan ligneux. Le dispositif expérimental mis au point au laboratoire permet de mesurer le module d'Young et le coefficient de Poisson sur des échantillons de faible taille. Dans ce travail, nous avons distingué quatre types d'éléments constitutifs du plan ligneux du chêne que nous nous sommes appliqués à isoler. Des micro-éprouvettes ont ainsi été usinées dans le rayon ligneux, le bois initial, le parenchyme longitudinal et les zones de fibre. Le rayon ligneux se révèle être la structure la plus rigide du plan transverse. À l'opposé, la zone initiale poreuse montre des modules d'élasticité près de dix fois moins importants. Entre les deux, nous retrouvons les plages de fibre et de parenchyme longitudinal du bois final. Contrairement aux autres tissus, ces dernières semblent présenter une forte anisotropie transverse $\left(E_{R} / E_{T} \approx 2\right)$. (@ Inra/Elsevier, Paris.)

propriétés élastiques / anatomie / chêne / microscopique / mesures

\section{Introduction}

À l'échelle macroscopique, le comportement mécanique du bois présente certains caractères bien reproductibles d'une essence à l'autre ou d'un arbre à l'autre au sein de la même essence. En particulier, la relation d'ordre entre les directions matérielles est souvent respectée : la direction longitudinale présente la valeur la plus élevée, d'un ordre de grandeur, vient ensuite la direction radiale puis la direction tangentielle. Par ailleurs, la densité à elle seule permet une évaluation correcte de ces différentes valeurs $[7,11]$. De ce point

\footnotetext{
* Correspondence and reprints

E-mail: perre@engref.fr
} 
de vue, les propriétés élastiques sont plus «stables» que d'autres propriétés physiques du bois. Des mesures récentes ont montré que la diffusivité massique du bois, par exemple, se prête beaucoup moins à une prédiction macroscopique [22]. La prédiction du retrait est également problématique.

Cependant, même avec de bonnes corrélations, la prédiction des propriétés mécaniques à partir de grandeurs macroscopiques présente une plage d'incertitude telle que la valeur peut aller du simple au double. Le rapport d'anisotropie, en particulier, est assez mal prédit.

En fait, le comportement mécanique du bois résulte directement de l'organisation structurale de sa matière ligneuse. Certains de ces caractères se retrouvent sur toutes les essences. Par exemple, les cellules sont toujours placées préférentiellement selon la direction longitudinale. Mais chaque essence est caractérisée par son plan ligneux et chaque individu présente une expression particulière de ce plan ligneux. En conséquence, il est légitime de rechercher les raisons de la variabilité à l'échelle anatomique.

De nombreux travaux explorant cette piste sont proposés dans la littérature. L'explication du rapport d'anisotropie, qui peut varier sensiblement pour des bois de même nature ayant la même densité, occupe à elle seule de nombreux chercheurs. En raison de leur plan ligneux assez simple, ils se sont plus particulièrement intéressés aux résineux. Les approches théoriques sont les plus nombreuses. L'objectif est de proposer des modèles du comportement mécanique de la structure anatomique qui représentent au mieux le milieu réel. Ils sont souvent de nature analytique. Le milieu est alors représenté par une " cellule moyenne " (paroi, trachéide ou accroissement annuel) caractérisée par certains paramètres géométriques $[5,6,12,13]$.

Plus récemment, les techniques d'analyse d'image, aidées par une résolution numérique de la solution analytique, ont permis de s'approcher un peu plus de la structure réelle [9]. En résolvant les problèmes d'homogénéisation sur un maillage d'éléments finis construit à partir d'images réelles, Farruggia [3] a montré l'importance de la forme et de l'agencement des cellules. Il est alors envisageable de prédire, par des calculs numériques réalisés sur des images, un certain nombre de propriétés prenant en considération la structure poreuse du matériau.

Cependant, toutes ces approches théoriques ont besoin de s'alimenter de valeurs mesurées. La difficulté réside dans l'échelle d'investigation requise : en dessous du millimètre, et parfois jusqu'au micromètre. Les techniques sont donc complexes, délicates à mettre en œuvre, et parfois difficile à interpréter [15-17, 21]. En particu- lier, afin d'éviter toute perturbation, le champ de déformation devrait se mesurer sans contact, ce qui n'est pas toujours le cas.

Par rapport aux résineux, le chêne pourrait se placer à l'autre extrémité de l'échelle de complexité du plan ligneux. Matériau composite fort complexe, il est fortement hétérogène par la diversité des éléments qui le composent, la variabilité de leur agencement au sein d'un accroissement annuel et leur propriétés spécifiques.

Si plusieurs modèles théoriques se proposent d'expliquer le comportement du bois massif en fonction de paramètres tels que la proportion de bois initial, de bois final ou de rayon ligneux $[1,2,8]$, ils se sont heurtés à la méconnaissance des propriétés propres à chacun de ces constituants.

Dans le cas du Chêne, où les constituants ont une forte densité, les propriétés de la paroi jouent un rôle déterminant, ce qui peut masquer l'effet de l'agencement cellulaire. La prédiction à l'aide de modèle théorique est alors sujette à plus de cautions : le recours à l'expérience est inévitable.

Cet article est consacré à la caractérisation mécanique des certains éléments du plan ligneux de chêne. Le principe expérimental est le même que celui qui fut mis au point sur des résineux [4]. En revanche, la préparation des échantillons est encore plus délicate. Du point de vue expérimental, il n'est pas imaginable d'accéder aux propriétés des différents composants à partir d'un même échantillon de bois. Aussi notre ambition se limitera-telle à tenter de mesurer le maximum de propriétés sur des échantillons de provenance différentes (nous entendons ici par provenance des arbres différents ou des positions différentes dans l'arbre). La zone de prélèvement est choisie en fonction de son expression particulière du plan ligneux, afin de permettre le prélèvement d'un échantillon «homogène» pour un élément particulier du plan ligneux.

\section{Matériel et méthode}

\subsection{Machine d'essais micromécaniques}

La méthode expérimentale utilisée dans ce travail est celle qui fut mis au point à l'Équipe produits forestiers du laboratoire de recherches en sciences forestières de l'Engref [18]. Elle a pour base une micromachine d'essais (figure 1). Elle permet d'effectuer des tests en traction sur des éprouvettes de faibles dimensions : une platine de déplacement permet de solliciter l'échantillon alors qu'une cellule de force restitue la valeur de la charge appliquée. L'ensemble est installé sous un microscope optique (figure 2) équipé d'une caméra pilotée par ordi- 


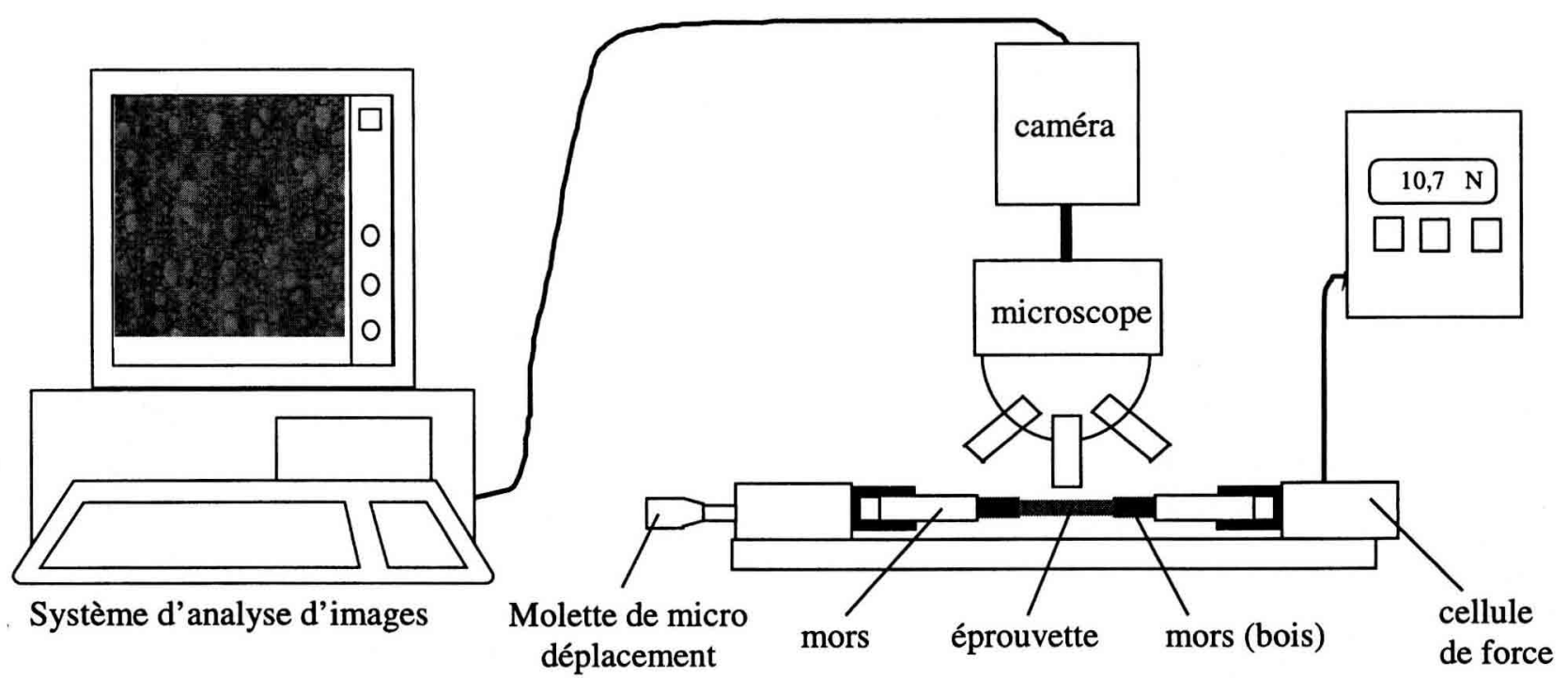

Figure 1. Système expérimental d'essai mécanique sous microscope développé à L’Engref. La comparaison d'images avant et après sollicitation permet de calculer le champ de déformation alors que le capteur de force permet d'accéder à la contrainte correspondante.

nateur. Un logiciel d'analyse d'image permet l'acquisition de vues qui serviront au calcul du champ de déformation.

Pour des échantillons dont les dimensions n'excèdent pas quelques millimètres dans la longueur et quelques centaines de micromètres dans la section, il incombe à l'expérimentateur d'accéder au champ de déformation sans contact. Une méthode utilisant l'image comme source d'information pour accéder au tenseur de déformation de l'éprouvette a été mise au point au laboratoire. Son principe repose sur la comparaison de la distribution des niveaux de gris des pixels entre l'image déformée et l'image de référence prise avant sollicitation. Le calcul permet d'accéder à trois composantes du tenseur de déformation dans le plan RT: $\varepsilon_{\mathrm{xx}}$, $\varepsilon_{\mathrm{yy}}$ et $\varepsilon_{\mathrm{xy}}$ ( $x$ étant la direction de la traction et $y$ la direction orthogonale dans le plan de l'image) supposé uniforme dans la fenêtre d'exploration. Le tenseur des contraintes, lui-même supposé uniforme dans la section, est réduit à une seule composante non nulle:

$$
\sigma_{x x}=\frac{F}{S}
$$

F est la force appliquée et $S$ la section de l'échantillon

Les deux composantes $\varepsilon_{\mathrm{xx}}$ et $\varepsilon_{\mathrm{yy}}$ mesurées en fonction de la charge $F$ permettent de tracer les courbes déformation-contrainte. En exprimant la loi de comportement élastique dans la cas d'une traction unidirectionnelle, la

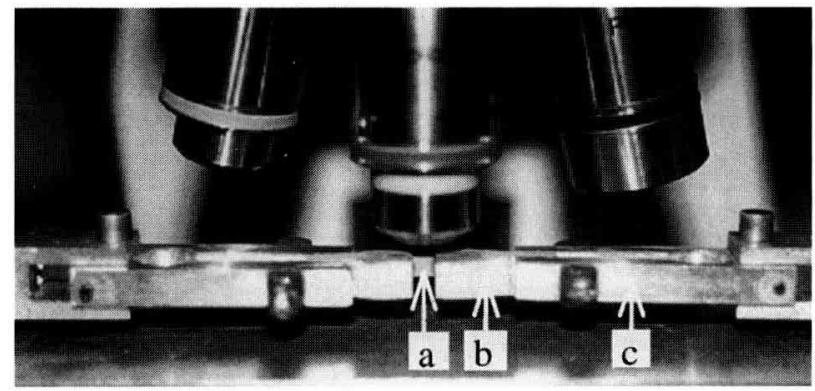

Figure 2. Micro machine d'essai placée sous microscope. L'éprouvette de traction (a) est collée dans des mors en alisier (b) eux même serrés dans des machoires métalliques (c).

valeur du module d'Young $\mathrm{E}_{\mathrm{x}}$ dans la direction de la sollicitation et celle du coefficient de Poisson, $\varepsilon_{\mathrm{xy}}$ sont données par:

$$
\sigma_{x x}=E_{x} \varepsilon_{x x} \text { et } \varepsilon_{y y}=-v_{x y} \varepsilon_{x x}
$$

La valeur de la déformation en cisaillement $\varepsilon_{x y}$ est utilisée afin de vérifier, a posteriori, que la traction a bien été réalisée selon les axes principaux du matériau. Par ailleurs, seuls les points situés dans la zone élastique linéaire sont pris en considération. À cette fin, les der- 
nières images sont éliminées lors de l'analyse des courbes expérimentales. Le calcul de chaque point de la courbe, effectué par comparaison de deux images, nécessite environ 2 min sur un PC à base de Pentium II.

Après avoir appliqué une précharge de quelques newtons, une première prise de vue de l'échantillon est réalisée. Elle constituera l'image de référence. La fenêtre d'exploration est choisie en fonction de la section de l'éprouvette afin de se situer dans la partie centrale de la face observée. Le grossissement est adapté à la taille et la structure anatomique de chaque échantillon. En général, l'objectif \pm 10 est utilisé. Il permet de cadrer un champ de mesure correspondant à un carré de $700 \mu \mathrm{m}$ d'arête pour une image numérisée de 256 par 256 pixels. À chaque incrément de chargement, une nouvelle image est mémorisée et la force appliquée est notée. L'essai prend fin avec la rupture de l'échantillon ou lorsque la valeur maximale supportée par la cellule de force est atteinte $(100 \mathrm{~N})$.

Une macrocommande a été écrite sur le logiciel de traitement d'images afin d'automatiser la numérotation et la mémorisation des images. Ceci permet de réduire le laps de temps entre les différentes acquisitions et de diminuer les effets dus au phénomène de relaxation des contraintes.

La qualité de l'image étant un paramètre important pour le calcul ultérieur du champ de déformation, le bruit propre à la caméra est largement atténué en intégrant le signal sur une trentaine d'images durant l'acquisition.

\section{2. Échantillonnage et préparation des éprouvettes}

La préparation d'un essai mécanique est la phase la plus longue et la plus délicate du processus. Elle se décompose en deux parties : la sélection de l'échantillon et la préparation de l'éprouvette de traction.

Le plan ligneux du chêne est extrêmement complexe par son hétérogénéité. Au sein d'un accroissement annuel, nous distinguons quatre zones, discernables à l'œil nu : le gros rayon ligneux, bien différencié par l'orientation de ses cellules dans la direction radiale, la zone initiale poreuse constituée essentiellement de gros vaisseaux et de parenchyme longitudinal et le bois final dans lequel nous séparons plages de fibre et plages de parenchyme contenant des petits vaisseaux (figure 3). S'il est à noter que l'on trouve quelques cellules de parenchyme longitudinal dans des zones de fibre, et réciproquement, nous garderons dans la suite du texte, par abus de langage, les appellations générales « plage de fibre » et «plage de parenchyme ». Nous intéressant ici aux propriétés mécaniques dans le plan transverse, cette distinction nous amène donc à envisager huit types d'essais mécaniques. Chacun d'eux nous conduit à la recherche de cernes particuliers permettant l'isolement d'une plage de tissu de taille suffisamment importante

a)
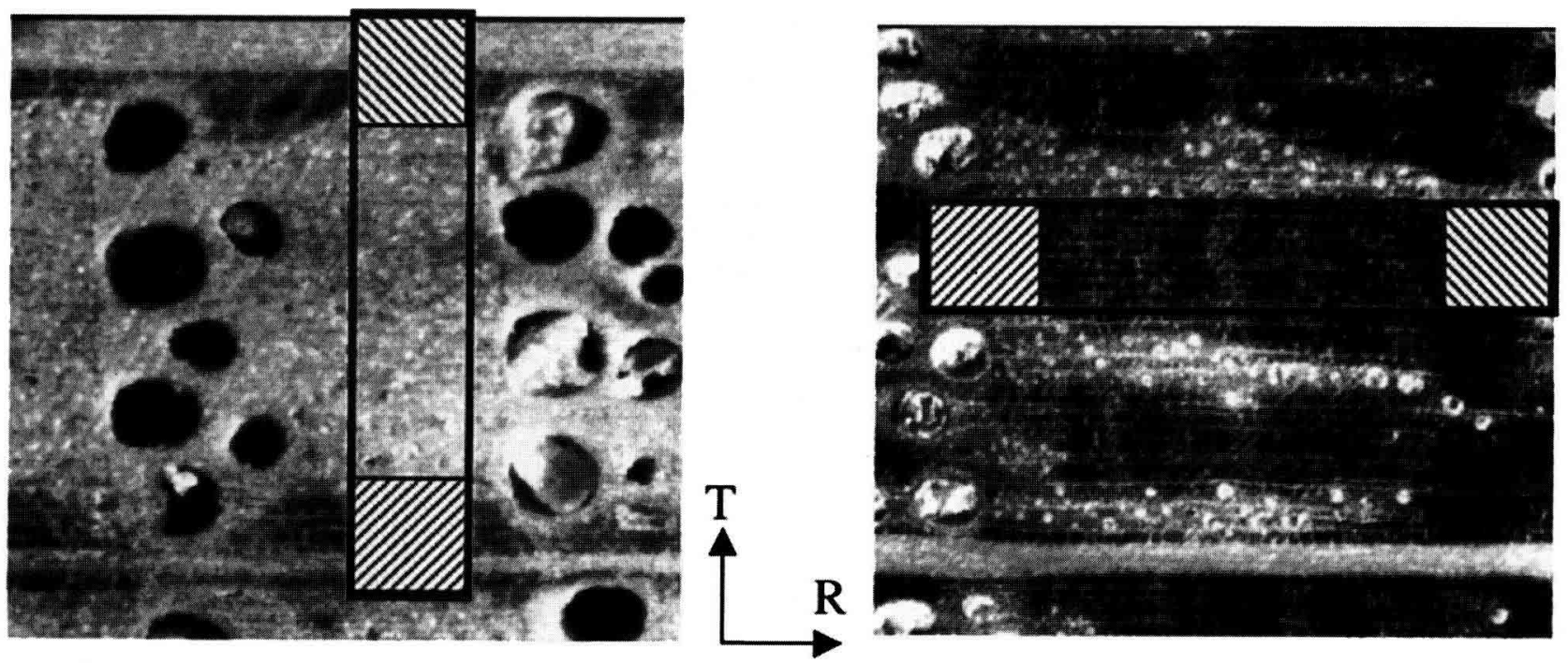

Figure 3. a. Échantillonnage d'une zone de parenchyme et petits vaisseaux destinée à un essai mécanique de traction dans la direction tangentielle. b. Échantillonnage d'une zone fibre destinée à un essai de traction dans la direction radiale. 
pour pouvoir y usiner une éprouvette. Pour un essai de traction tangentielle dans une plage de parenchyme, on recherchera une zone à forts accroissements annuels sans développement de fibres et sans gros rayon ligneux (figure $3 a$ ). Par opposition, un essai de traction sur la zone de fibre dans la direction radiale sera effectué dans une zone à très faible proportion de parenchyme figure $3 b)$.

Cette recherche de cernes d'accroissement aussi particuliers ne peut malheureusement se faire qu'au détriment de l'homogénéité de l'échantillonnage. Aussi devons nous avoir à l'esprit que les échantillons peuvent provenir de cernes ou d'arbres différents. Les propriétés mécaniques à l'échelle cellulaire, c'est-à-dire les propriétés même des parois sont donc vraisemblablement différentes en raison de leur composition chimique, de l'arrangement des différentes couches qui les composent ainsi que de la structure même de ces dernières (angle des microfibrilles, différence de cohésion entre les couches $\mathrm{S} 1$ et $\mathrm{S} 2 \ldots$... . Cette remarque est en grande partie responsable de la forte variabilité attendue, et obtenue, dans les résultats.
L'échantillon est obtenu par ponçages successifs à l'aide de disques abrasifs de plus en plus fins collés sur des plateaux de verre. Ce polissage a pour objectif d'obtenir une éprouvette de section constante et homogène, c'est-à-dire constituée d'un seul type de tissu. La section et la longueur de l'échantillon dépendent du type de tissu considéré, l'épaisseur n'étant souvent que de quelques centaines de micromètres. Cette étape longue et délicate conditionne très fortement la qualité des résultats de l'expérience et nécessite de l'ordre d'une journée de préparation. La difficulté et le temps de préparation sont des éléments qui restreignent considérablement le nombre d'essais effectués. Enfin, afin de garantir une certaine stabilité hydrique, les essais sont réalisés pendant la période hivernale dans une pièce chauffée. Ainsi les échantillons ont-ils une teneur en eau comprise entre 6 et $8 \%$.

Après polissage, les éprouvettes sont collées pendant une nuit à l'intérieur de fentes, préparées par un sciage fin, dans des mors en bois préalablement fixés sur la machine d'essai (figure 4). En raison de la faible taille des éprouvettes, cette opération est très délicate. La qua-
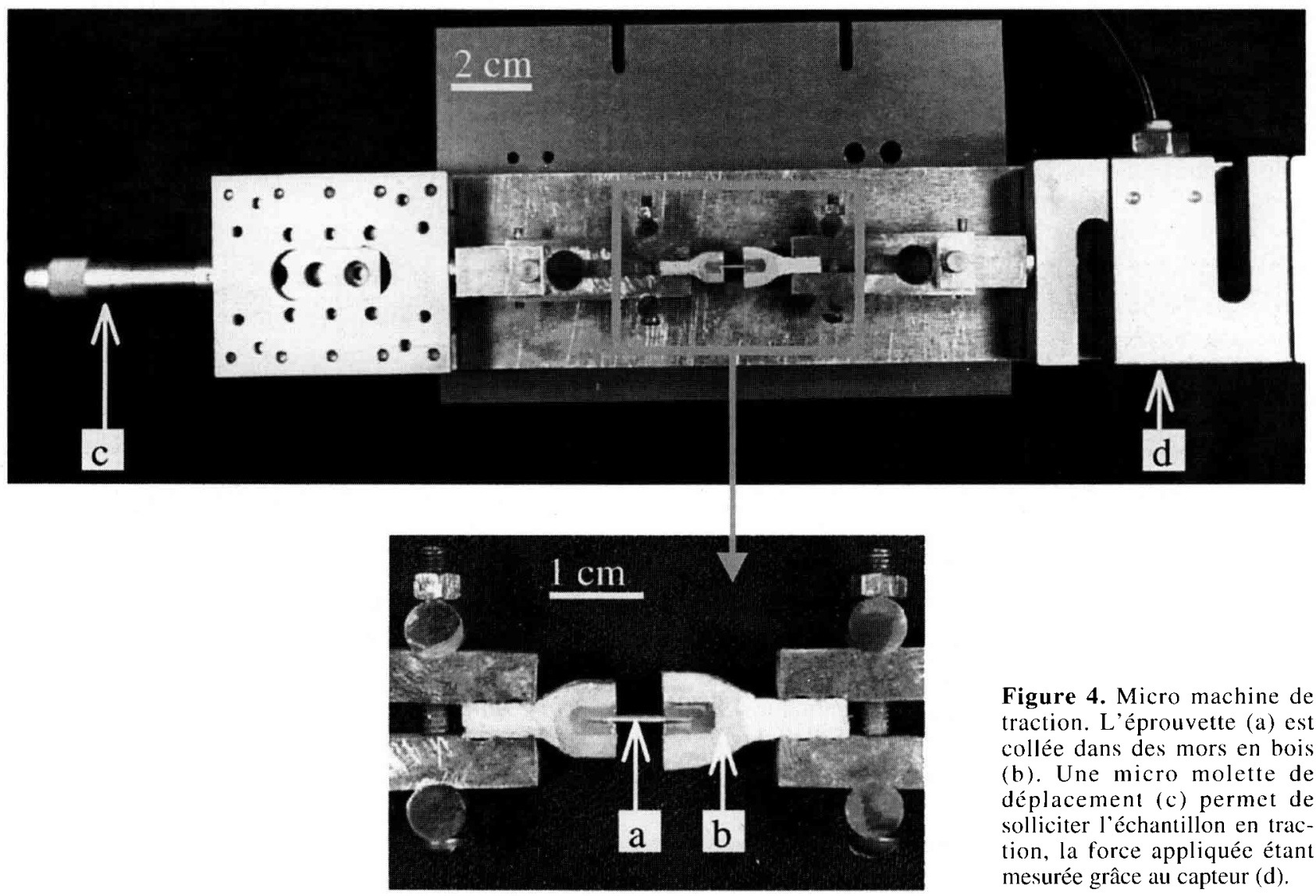

Figure 4. Micro machine de traction. L'éprouvette (a) est collée dans des mors en bois (b). Une micro molette de déplacement (c) permet de solliciter l'échantillon en traction, la force appliquéc étant mesurée grâce au capteur (d). 
lité du positionnement de d'échantillon étant primordiale pour la suite de l'expérience, il est parfois nécessaire de réaliser cette opération sous microscope.

\subsection{Estimations des erreurs expérimentales}

La première source d'erreur provient de l'usinage de l'échantillon. La précision de ses dimensions, en particulier la régularité de sa section, est un facteur essentiel pour pouvoir appliquer les formules simples énoncées plus haut. Des mesures sont donc réalisées en différents points à l'aide d'un pied à coulisse dont les indications sont précises au $1 / 100 \mathrm{~mm}$ (figure 5). L'usinage est achevé lorsque toutes les valeurs mesurées sont égales à $10 \mu \mathrm{m}$ près pour une section voisine de $1 \mathrm{~mm}$ par $2 \mathrm{~mm}$. Il est donc possible de chiffrer l'erreur sur la section de l'éprouvette à environ $5 \%$.

Durant l'essai mécanique, on peut observer, après chaque nouvel incrément de force, une relaxation de l'échantillon. Celle-ci varie en fonction de la nature de l'éprouvette et augmente au cours de l'essai mais ne dépasse pas $5 \%$ de la valeur mesurée entre deux prises de vue.

Nous avons déterminé l'erreur due au calcul du champ de déformation. Le nombre d'intégrations réalisées lors de l'acquisition joue ici un rôle important (figure 6). Cependant, un compromis doit être trouvé entre le temps d'intégration et les problèmes de relaxation cités ci-dessus. En pratique, les conditions expérimentales choisies sont telles que la précision reste de l'ordre de $10^{-4}$.

Les propriétés mécaniques sont calculées à partir de droites de régressions. L'essai est validé lorsque les coefficients de corrélation sont assez grands pour que les valeurs calculées soient considérées comme significatives. Pour le module d'Young, la valeur limite a été fixée à $R^{2}=0,95$. Les valeurs de déformation relatives au coefficient de Poisson étant généralement plus faibles, et donc plus difficiles à mesurer, nous avons été moins exigeants sur cette corrélation. Les figures 7 et 8 montrent deux exemples dont les valeurs sont jugées significatives pour les deux paramètres calculés.

Le premier essai est caractéristique d'un excellent essai : les points sont pratiquement placés sur une droite. Le second a été choisi pour illustrer le minimum requis pour que l'essai soit déclaré acceptable. Ici, l'alignement des points reste bon, mais la dispersion des points est bien perceptible.

Deux méthodes sont a priori possibles pour obtenir le coefficient de Poisson :

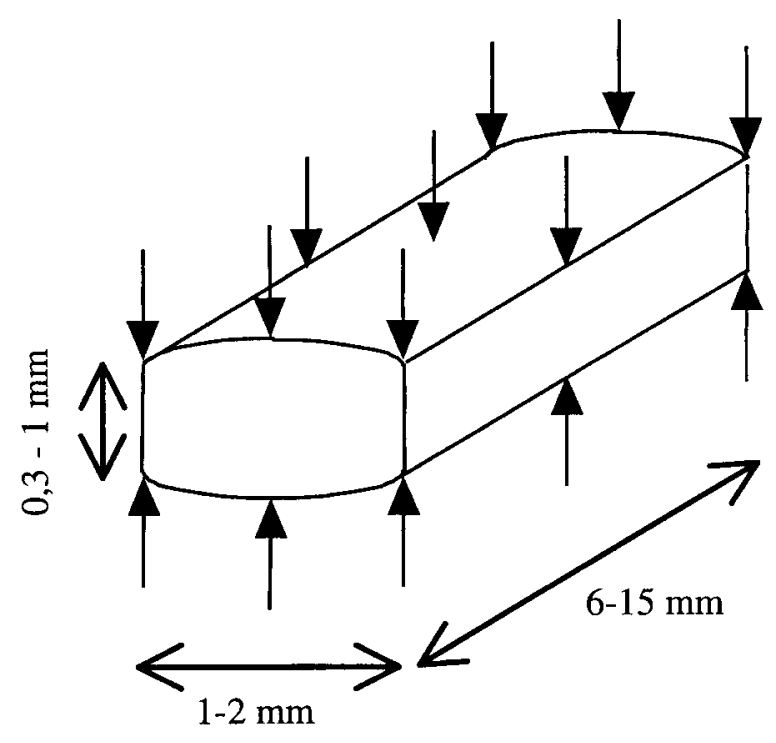

Figure 5. Points de mesures permettant le contrôle du parallélisme des faces de l'éprouvette (les valeurs sont données à titre indicatif)

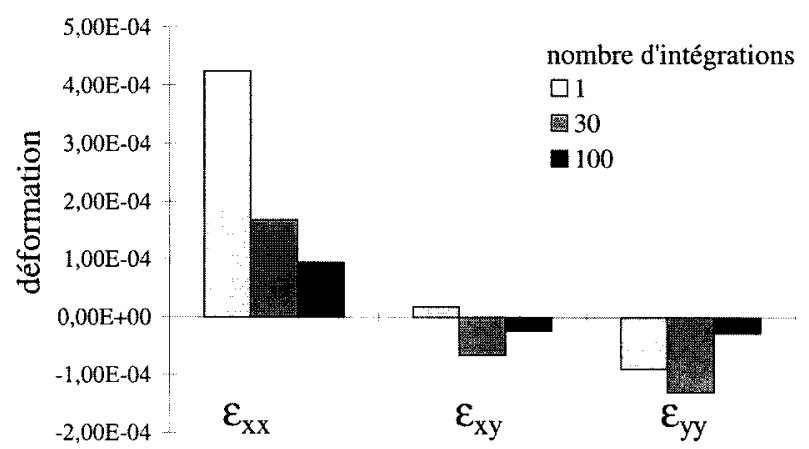

Figure 6. Influence du nombre d'intégrations réalisées lors de l'acquisition sur la précision de la mesure du champ de déformation. Comparaison de deux images $256 \times 256$ translatées.

- Utiliser l'opposé du rapport des pentes calculées sur les deux courbes expérimentales, $\varepsilon_{y y}=\mathrm{f}\left(\sigma_{x x}\right)$ et $\varepsilon_{x x}=\mathrm{f}\left(\sigma_{x x}\right)$.

- Calculer la pente de la courbe $\varepsilon_{y y}=\mathrm{f}\left(\varepsilon_{x x}\right)$.

La première méthode est facile à mettre en œuvre et est utilisable même avec des essais médiocres. 


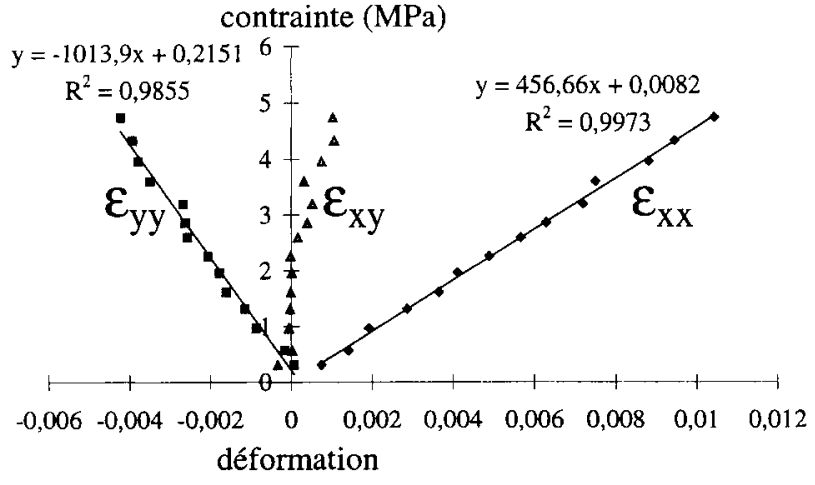

a

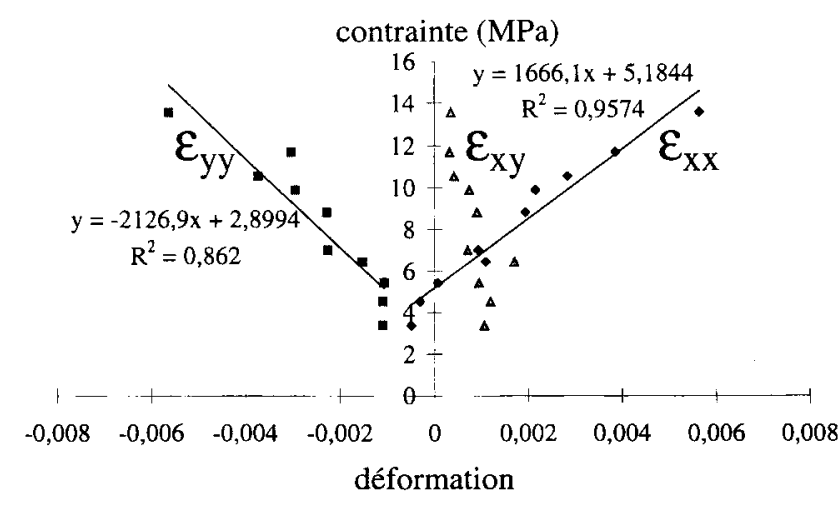

b

Figure 7. Calcul du module d'Young par la relation contrainte-déformation, $\varepsilon=\mathrm{f}(\varepsilon)$, lors d'un essai de traction dans le plan transverse : les indices xx et yy correspondent respectivement à la direction de sollicitation de l'échantillon et à son orthogonale. Chaque point expérimental est le résultat de la comparaison de la distribution des niveaux de gris d'une image avec ceux de l'image de référence (éprouvette non chargée) $-\mathrm{a}$ : cas d'une zone initiale poreuse. $\mathrm{E}_{\mathrm{R}}=456 \mathrm{MPa}-\mathrm{b}:$ cas d'une plage de parenchyme. $\mathrm{E}_{\mathrm{R}}=$ $1666 \mathrm{Ma}$.

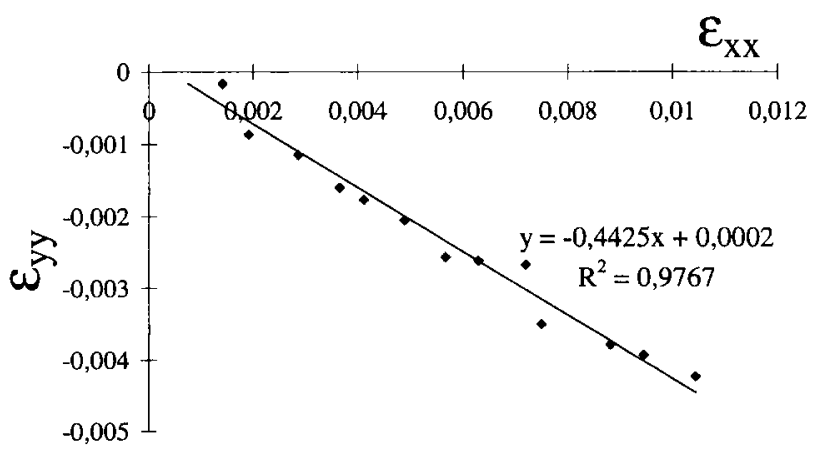

a

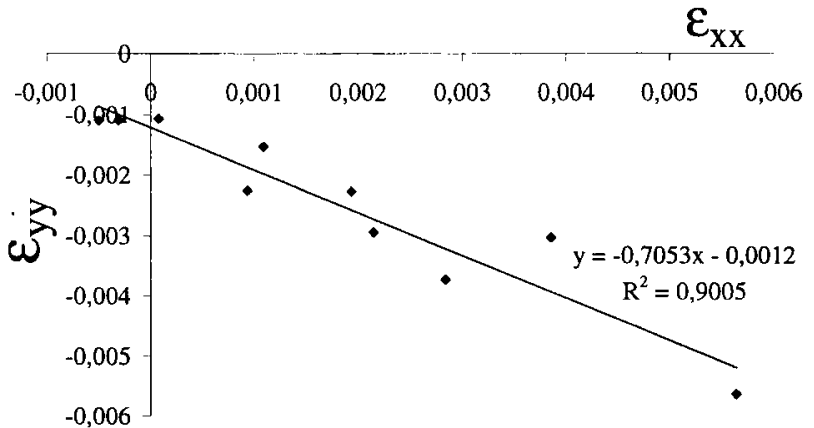

b

Figure 8. Calcul du coefficient de Poisson par la relation $\varepsilon_{\mathrm{yy}}=\mathrm{f}\left(\varepsilon_{\mathrm{xx}}\right)$.- a : cas d'une zone initiale poreuse. $\varepsilon_{\mathrm{RT}}=0.44-\mathrm{b}:$ cas d'une plage de parenchyme. $\varepsilon_{\mathrm{RT}}=0.70$ (traction radiale).

Cependant, nous avons préféré utiliser la deuxième méthode, dont le principe repose sur la définition même du coefficient de Poisson. Par ailleurs, en traçant la courbe correspondante, à partir de la valeur de $\mathrm{r}^{2}$, il est possible d'utiliser le même critère de validation (figure 8).

\section{Résultats}

Les contraintes liées à l'échantillonnage limitent fortement le nombre d'expériences : nous avons effectué une quarantaine d'essais mécaniques au cours de deux campagnes. 33 ont été jugés concluants. Les figures 9 et 10 résument l'ensemble des mesures réalisées. Les déformations dues à l'effet Poisson étant plus petites que celles nécessaires au calcul du module d'Young, il n'est pas toujours possible de calculer le coefficient correspondant. Seuls une vingtaine de points apparaissent donc sur la figure 10.

$\mathrm{Si}$, pour certains éléments, les résultats semblent relativement reproductibles (zone de parenchyme, de fibre), d'autres montrent une variabilité plus importante. Le retour à une description anatomique sommaire du matériau permet de formuler les hypothèses explicatives de 


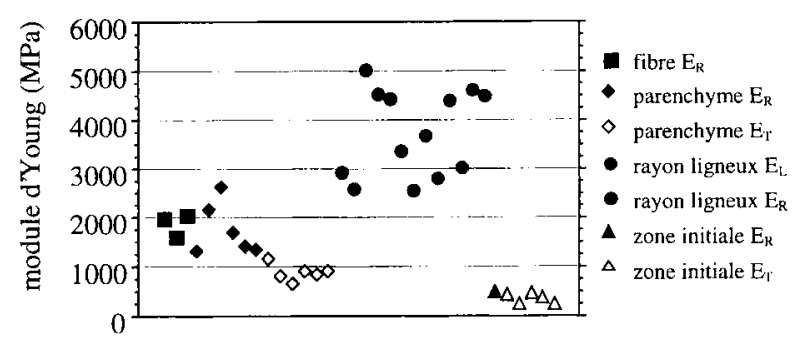

Figure 9. Graphique récapitulatif des résultats obtenus sur les modules d'Young mesurés par traction de plages de tissu isolćes.

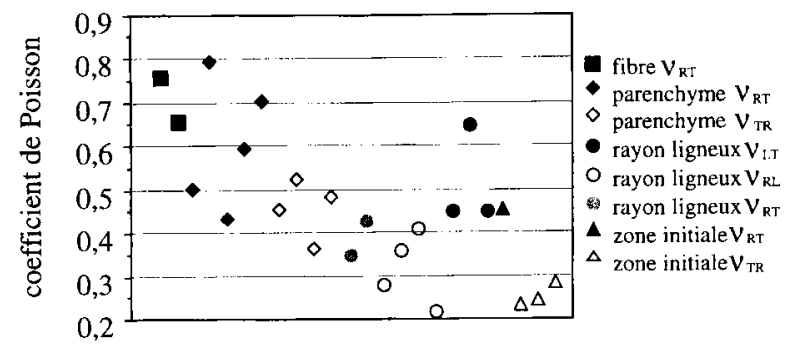

Figure 10. Graphique récapitulatif des résultats obtenus sur les coefficients de Poisson mesurés par traction de plages de tissu isolées. son comportement et de la dispersion éventuelle des résultats. Les valeurs indiquées sont des moyennes suivies de leur écart-type.

\subsection{La zone initiale poreuse}

Il est relativement aisé d'usiner des éprouvettes afin de les solliciter dans la direction tangentielle. Il est pour cela nécessaire de trouver un spécimen comportant des gros rayons ligneux suffisamment espacés dans cette direction, ceci conditionnant la longueur finale de l'échantillon. Cinq essais ont été réalisés et leur reproductibilité nous permet d'accorder une bonne confiance dans ces mesures. Les résultats donnent un module d'élasticité $\mathrm{E}_{\mathrm{T}}$ toujours très faible d'environ $320 \pm 85$ $\mathrm{MPa}$ et un coefficient de Poisson $v_{\mathrm{TR}}$ d'environ $0,25 \pm$ 0,02 .

En revanche, il est utopique de vouloir réaliser un essai de traction radiale dans un seul cerne. S'il fut envisagé de travailler sur un échantillon comportant une alternance de bois d'été et de bois de printemps en n'explorant que cette dernière zone lors du calcul des composantes du champ de déformation, le couplage mécanique entre ces deux parties se révèle trop important pour pouvoir ensuite analyser les résultats. Nous avons donc cherché un spécimen où l'accroissement annuel reste, pendant plusieurs années, si fin que le bois final est inexistant (figure 11). La mesure, qui n'a pas pu
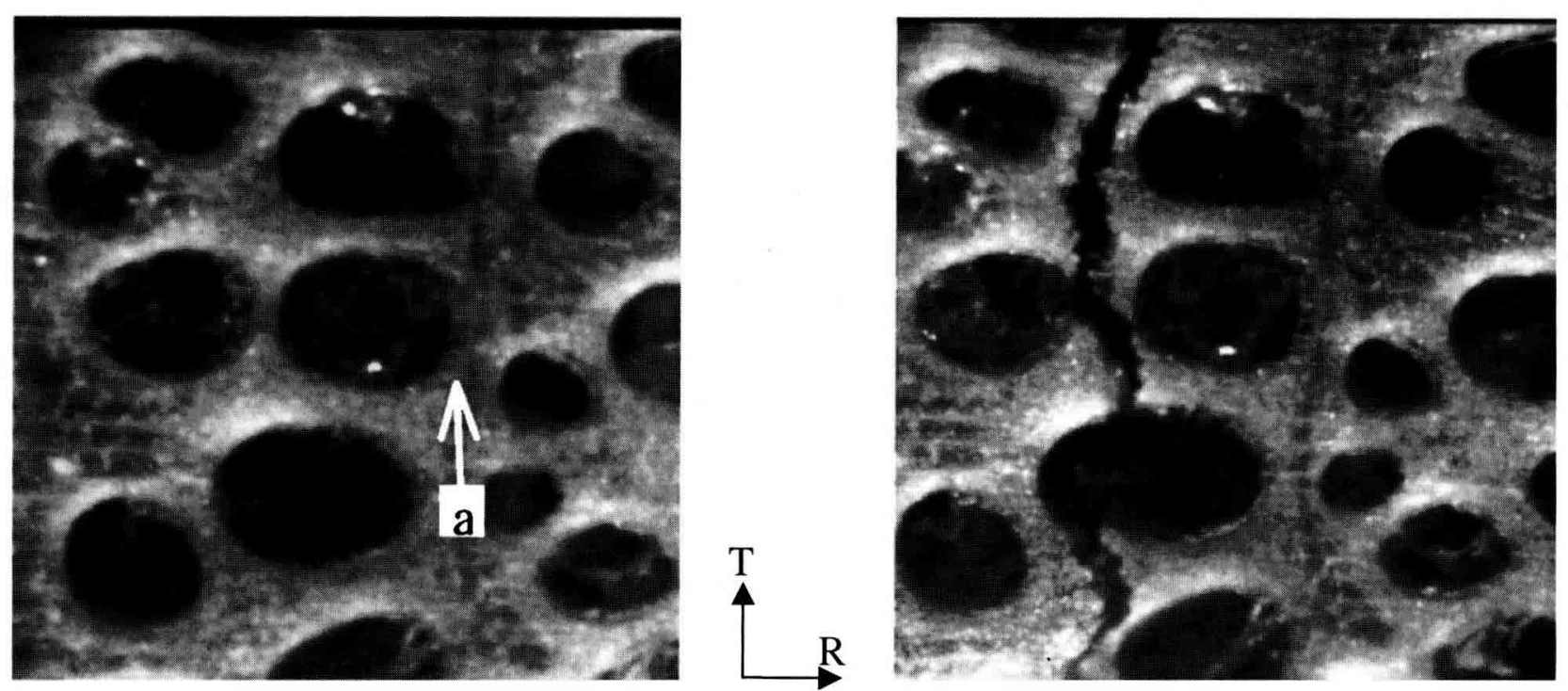

Figure 11. Zone initiale poreuse. La zone de bois final quasiment inexistante (a) permet de réaliser un essai en traction radiale. Éprouvette avant sollicitation (a) et après rupture (b). 
être répétée, donne un module d'élasticité encore très faible $\left(E_{R}=450 \mathrm{MPa}\right)$ mais supérieur à toutes les rigidités mesurées jusqu'à lors pour la même zone dans la direction tangentielle. Un fort coefficient de Poisson $v_{R T}$, d'une valeur de 0,45 , a pu être mesuré. Nous retrouvons quasiment ici une propriété de base liée à la symétrie du tenseur des rigidités :

$$
\frac{E_{R}}{v_{R T}}=\frac{E_{T}}{v_{T R}}
$$

Cependant, devant l'unicité de ce résultat, et en dépit de la faible valeur du cisaillement pourtant caractéristique d'un essai concluant (figure $7 a$ ), nous nous garderons d'avancer des conclusions hâtives sur les propriétés mécaniques de la zone initiale poreuse dans la direction radiale.

Lors d'observations anatomiques, on constate que la forme et la disposition des différents éléments qui composent la zone initiale poreuse varient considérablement d'un échantillon à l'autre. Il est donc très difficile de se prononcer sur une quelconque anisotropie marquée ou non. En particulier, les gros vaisseaux jouent un rôle primordial. Leur nombre, leur taille et leur forme influencent considérablement sur la nature des résultats. Cependant, nous constaterons qu'ils sont généralement plus allongés dans la direction radiale que dans la direction tangentielle. De plus, la présence des petits rayons ligneux unisériés tend à rigidifier le matériau dans cette direction. Ces deux remarques concourent à renforcer la valeur du module d'Young $\mathrm{E}_{\mathrm{R}}$ (essai en traction radiale) et à diminuer celle du coefficient de Poisson $\mathrm{n}_{\mathrm{TR}}{ }^{1}$.

\subsection{Les plages de fibre}

Les plages de fibre sont les zones les plus denses du plan ligneux du chêne $\left(\approx 1200 \mathrm{~kg} \mathrm{~m}^{-3}\right)$. Elles ont essentiellement un rôle de soutien mécanique dans l'arbre. Si expérimentalement il est possible d'usiner des éprouvettes dans la direction radiale, cela n'est malheureusement pas le cas dans la direction tangentielle en raison de l'agencement de ces plages en bandes radiales au sein de l'accroissement annuel (figure $3 b$ ). Quatre essais, dont trois concluants, ont montré des résultats très forts, tant pour le module d'Young $\left(E_{R}=1900 \pm 175 \mathrm{MPa}\right)$ que pour le coefficient de Poisson $\left(\mathrm{n}_{\mathrm{RT}}=0,71 \pm 0,05\right)$.

$\mathrm{Si}$ aucune valeur ne peut être avancée dans la direction tangentielle, l'observation anatomique nous ren-

\footnotetext{
${ }^{1}$ Rappel : ce paramètre quantifie la déformation radiale mesurée lors d'un essai en traction tangentielle
}
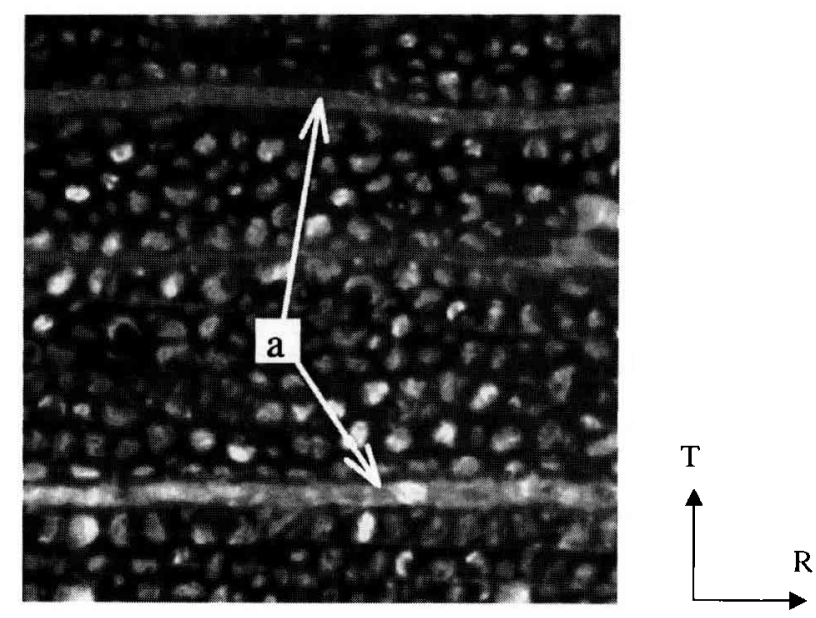

Figure 12. Plage de fibre traversée de petits rayons ligneux unisériés (a) dans la direction radiale.

seigne sur la disposition des cellules qui peut être tout à fait irrégulière ou légèrement orientée dans la direction radiale [10] mais jamais de façon très prononcée. Cependant, la présence de petits rayons ligneux unisériés confère au matériau une légère prédominance de la direction radiale (figure 125). Nous pouvons donc présumer que l'éventuelle anisotropie du matériau ne pourra pas être très marquée.

\subsection{Les gros rayons ligneux}

Les cellules de parenchyme constitutives des rayons ligneux sont allongées dans la direction radiale de l'arbre. Leur organisation particulière en un plan longitudinal-radial peu épais dans la direction tangentielle (figure 13) ne permet pas l'échantillonnage d'éprouvettes dans cette direction. Par contre, en sélectionnant des spécimens à rayons très épais, sept essais ont pu être réalisés en traction radiale et six dans la direction longitudinale.

La géométrie particulière des rayons ligneux nous a conduit à observer la face RL pour le calcul du champ de déformation lors des essais en traction radiale. Dans cette configuration, nous avons accès au module d'Young $E_{R}$ et au coefficient de Poisson $n_{R L}$. Dans ce plan RL, les repères anatomiques ne sont pas suffisamment visibles pour calculer le champ de déformation (mauvais contraste entre vides et parois cellulaires). Nous traçons donc une grille sur la surface observée à l'aide d'une mine très fine (figure 13). Lors des essais en traction longitudinale, la face observée peut être LR ou 


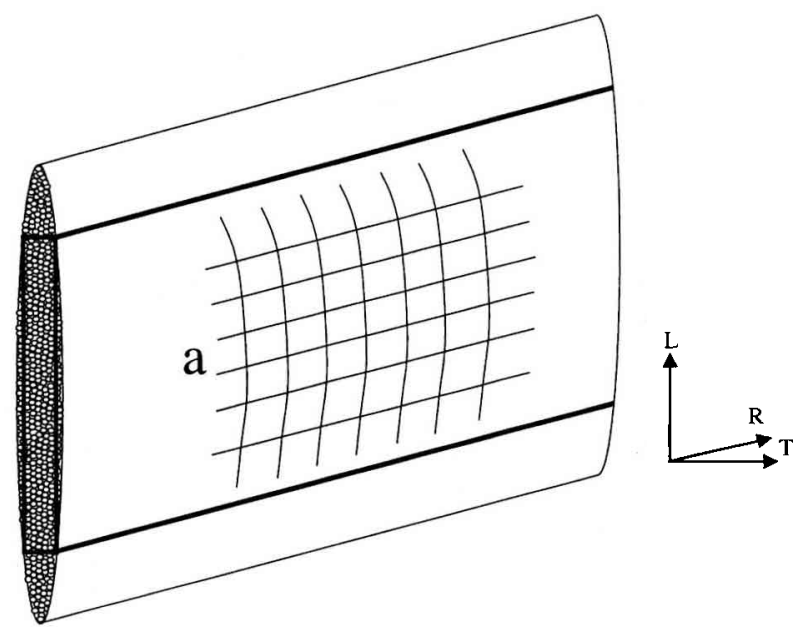

Figure 13. Découpe d'une éprouvette de traction dans un rayon ligneux - ici pour un essai en traction radiale, un réseau est tracé sur la face RL observée.

LT. Nous pouvons donc mesurer $E_{R}$ et $n_{L R}$, ou $n_{L T}$, suivant l'orientation de l'échantillon sur la machine d'essai.

Si les valeurs obtenues pour $E_{R}$, de l'ordre de $3600 \pm$ $900 \mathrm{MPa}$ (figure 9), sont les plus fortes qui aient pu être mesurées dans le plan transverse, il n'en demeure pas moins qu'elles sont nettement en dessous de ce que nous attendions. En effet, l'orientation des cellules (figure 13) tendrait à faire l'hypothèse, généralement retenue dans la littérature, que les propriétés radiales des rayons ligneux sont du même ordre de grandeur que celles du bois massif en longitudinal. Or les valeurs de module d'Young mesurées ici lors d'essais de traction longitudinale ne montrent pas d'anisotropie marquée entre les direction $\mathrm{R}$ et L. Deux hypothèses, déjà avancées par El Amri [2], peuvent être formulées pour expliquer ce résultat expérimental.

- La longueur des cellules de parenchyme est beaucoup plus faible dans les rayons ligneux que dans les autres types de tissu [10] limitant ainsi le caractère particulier du grand axe des cellules.

- Au sein de la paroi cellulaire, l'angle des microfibrilles est très élevé par rapport au grand axe [1].

Selon cette seconde hypothèse, les rigidités dans le plan TL, plan transverse des cellules, devraient être très fortes. C'est ce qui a pu être constaté lors des essais dans la direction longitudinale (figure 9) avec des valeurs mesurées pour le module d'Young $\left(E_{L}=3800 \pm 700\right.$ $\mathrm{MPa}$ ) près de deux fois supérieures à celles des plages de fibre, matériau pourtant plus dense et très rigide.
Pour les raisons évoquées précédemment, nous ne pouvons pas donner de valeurs dans la direction tangentielle et seule l'observation anatomique dans le plan TL peut nous renseigner sur une éventuelle anisotropie. Si l'organisation cellulaire semble ne privilégier aucune direction dans la partie centrale du rayon ligneux, on remarque un alignement progressif dans la direction longitudinale lorsque l'on se rapproche des bords avec une tendance générale à l'ovalisation des cellules selon cette direction. En conséquence, la rigidité serait donc plus faible dans la direction tangentielle.

En fonction de la face observée par la caméra, nous avons pu mesurer trois coefficients de Poisson : $\mathrm{n}_{\mathrm{LT}}=0,48 \pm 0,11, \mathrm{n}_{\mathrm{RT}}=0,34 \pm 0,05$ et $\mathbf{n}_{\mathrm{RL}}=0,32 \pm 0,06$.

\subsection{Les plages de parenchyme longitudinal de bois final}

Cette zone, constituée de cellules de parenchyme longitudinal et de petits vaisseaux, est moins dense que les plages de fibre $\left(\approx 800 \mathrm{~kg} \cdot \mathrm{m}^{-3}\right)$. Douze essais ont été validés ; six dans chaque direction. Les mesures mettent en évidence une forte anisotropie transverse, avec une valeur de module d'Young de $850 \pm 110 \mathrm{MPa}$ et $1700 \pm$ $450 \mathrm{MPa}$, respectivement dans les directions tangentielle et radiale. On observe une variabilité importante sur les résultats des coefficients de Poisson $\mathrm{n}_{\mathrm{RT}}=0,6 \pm 0,11$ et $\mathrm{n}_{\mathrm{TR}}=0,45 \pm 0,05$. Lors de l'observation de la zone de parenchyme, on constate que la structure est fortement conditionnée par la présence ou non de petits vaisseaux dont le nombre et la taille peuvent se révéler importants. Ils sont généralement alignés dans la direction radiale en semblant être guidés par les nombreux petit rayons ligneux unisériés (figure 14). L'organisation de ce tissu est donc nettement dépendante de ces deux paramètres qui confèrent à la direction radiale un rôle particulier. Cette structure explique aussi l'importante variabilité des résultats.

\section{Conclusion et perspectives}

Le travail présenté dans ce texte a permis de mesurer un grand nombre des rigidités des différents composants du plan ligneux du Chêne dans le plan transverse. L'usinage d'éprouvettes homogènes de taille suffisante pour réaliser les essais reste une difficulté majeure de cette partie expérimentale. La première conséquence directe est l'inaccessibilité de certaines valeurs (module d'Young tangentiel du rayon ligneux ou de la fibre).

L'impossibilité de travailler dans le même cerne, voire dans le même arbre est une autre contrainte impor- 

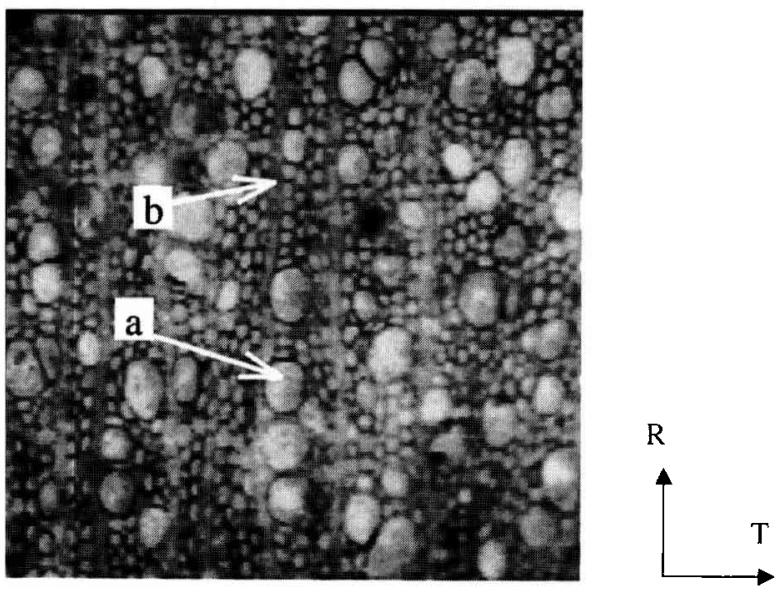

Figure 14. Zone de parenchyme contenant de nombreux petits vaisseaux (a) et rayons ligneux unisériés (b).

tante liée à la préparation de l'éprouvette. Cet aspect limite fortement la possibilité de maitriser une part de la variabilité du matériau. Dans ces conditions, il est évident que les valeurs mesurées ne peuvent être prises comme données absolues des propriétés du chêne. Elles permettent néanmoins de dégager des ordres de grandeur de propriétés d'éléments du plan ligneux inconnues jusqu'alors.

Le rayon ligneux se révèle être la structure la plus rigide du plan transverse. L'agencement des cellules, très courtes, de parenchyme radial qui le composent ne semble pas lui conférer de propriétés plus importantes en radial que dans le plan TL. À l'opposé, la zone initiale poreuse montre des modules d'élasticité près de dix fois moins importants. Le module radial de cette zone initiale semble légèrement plus fort que le module tangentiel.
Entre ces deux éléments, nous retrouvons les plages de fibre et de parenchyme du bois final. Contrairement aux autres tissus, ces dernières semblent présenter une forte anisotropie transverse $\mathrm{E}_{\mathrm{R}} / \mathrm{E}_{\mathrm{T}} \approx 2$ (tableau $I$ ).

Afin de mieux comprendre la relation entre l'organisation cellulaire des différents tissus et leurs propriétés mécaniques, il est prévu de réaliser des calculs numériques sur des images de la morphologie des éprouvettes de traction utilisées pour la présente étude.

Enfin, ces grandeurs élastiques mesurées à l'échelle microscopique sont le point de départ incontournable pour appliquer au matériau bois les techniques d'homogénéisation, bien connues dans le domaine des milieux poreux. Cette démarche, fortement développée dans notre laboratoire, propose une prédiction déterministe des propriétés du bois à partir de la morphologie anatomique [19]. Dans ce cadre, les propriétés mécaniques à l'échelle microscopique sont nécessaires à la prédiction des propriétés mécaniques à l'échelle macroscopique, mais également à celle du retrait. Elles sont également indispensables pour connaître le champ de microcontraintes généré par l'hétérogénéité du milieu poreux (fissuration de séchage, gélivure...).

\section{Références}

[1] Barkas W.W., Wood water relationships, VI. The influence of ray cells on the shrinkage of wood, Trans. Faraday Soc. 37 ( 1941 ) 535-547.

[2] El Amri F., Contribution à la modélisation élastique anisotrope du matériau bois - feuillus et résineux, thèse INPL, 1987.

[3] Farruggia F., Détermination du comportement élastique d'un ensemble de fibres de bois à partir de son organisation cellulaire et d'essais mécaniques sous microscope, thèse Engref, 1998.

Tableau I. Tableau récapitulatif des valeurs moyennes mesurées lors des essais de traction sur des plages de tissu isolées.

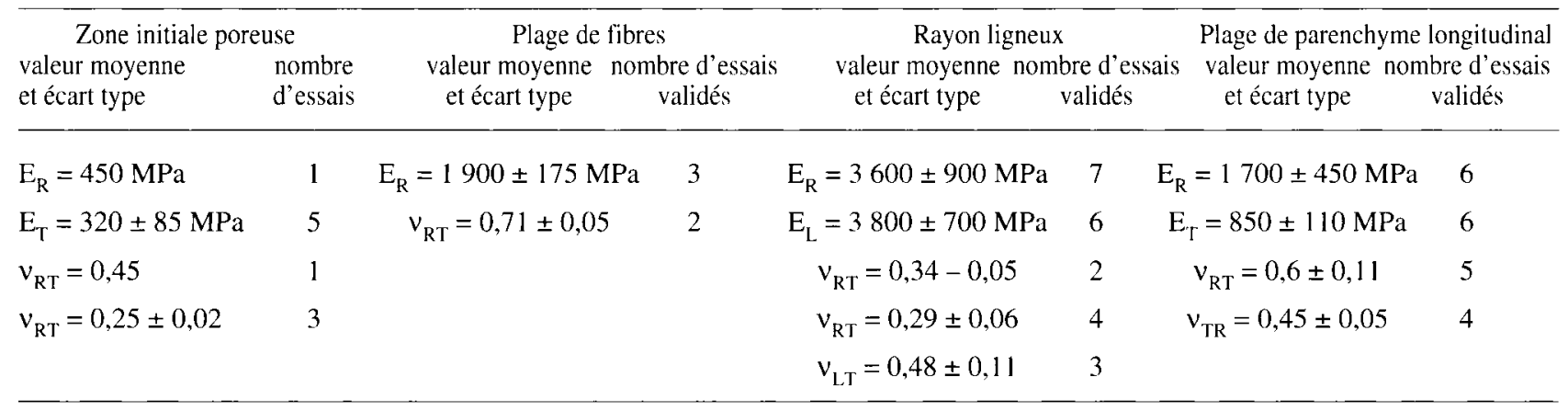


[4] Farruggia F., Perré P., Microscopic tensile tests in the transverse plane of earlywood and latewood parts of spruce, à paraître dans Wood Sci. Technol. (1999).

[5] Gibson L.J., Ashby, M.F., Cellular solids, Pergamon Press, 1988.

[6] Gillis P.P., Orthotropic Elastic Constants of Wood, Wood Sci. Technol. 6 (1972) 138-156.

[7] Guitard D., Mécanique du matériau bois et composites, Cepadues, Toulouse, France, 1987.

[8] Guitard D., Le bois matériau d'ingénierie, Arbolor, 1994, pp. 92-125.

[9] Harrington J.J., Booker R., Astley R.J., Modelling the elastic properties of softwood. Part I - The cell-wall lamellae. Part II - The cellular microstructure, Holz als Roh- und Werstoff 56 (1998) 37-50.

[10] Keller R., Le bois matériau d'ingénierie, Arbolor, 1994, pp. 14-52.

[11] Kollman F.P., Cote W.A., Principles of wood science and technology, Tome I, Springer Verlag, 1968.

[12] Koponen S., Torrati T., Kanerva P., Modelling longitudinal elastic and shrinkage properties of wood, Wood Sci. Technol. 23 (1989) 55-63.

[13] Koponen S., Torrati T., Kanerva P., Modelling elastic and shrinkage properties of wood based on cell structure, Wood Sci. Technol. 25 (1991) 25-32.

[14] Lahbabi R., Mesure par analyse d'image de paramètres microscopiques requis pour une prédiction déterministe des propriétés du bois, thèse Engref, 1995.
[15] Mott L., Shaler S.M., Groom L.H., A Technique to measure strain distributions in single wood pulp fibers, Wood Fiber Sci. 28 (1996) 429-437.

[16] Navi P., Rastogi P.K., Pittet V., Tolou A., Micromechanics of wood subjected to axial tension. Wood Sci. Technol. 29 (1995) 411-429.

[17] Oliver W.C., PharrR G.M., An improved technique for determining hardness and elastic modulus using load and displacement sensing indentation experiments, J. Matter. Res. 7 (1992) 1564-1583.

[18] Perré P., Lahbabil R., L'anatomie quantitative pour une approche déterministe des propriétés du matériaux bois. Rapport final de la convention de recherche DGER $n^{\circ} 91211$, 1995.

[19] Perré P., Image Analysis, Homogenization, Numerical Simulation and Experiment as Complementary Tools to Enlighten the Relationship between Wood Anatomy and Drying Behavior. Drying Technol. J. 15 (1997) 221 1-2238.

[20] Trenard Y., Gueneau P., Relation entre la structure anatomique et l'amplitude du retrait du bois, Holzforschung. Bd. 31 (1977) 194-200.

[21] Wimmer R., Lucas B. N, Tsui T. Y. et Oliver W.C., Longitudinal hardness and Young's modulus of spruce tracheid secondary walls using nanoindentation technique, Wood Sci. Technol. 31 (1997) 131-141.

[22] Zohoun S. - Détermination de la diffusivité massique dans le domaine hygroscopique du bois : comparaison des mesures en régimes permanent et transitoire, thèse INPL, Nancy, 1998. 